\title{
Integração Cultural na Escola Latino Americana de Agroecologia
}

\author{
La integración cultural en la Escuela Latinoamericana de Agroecología \\ Cultural Integration at the Latin American School of Agroecology
}

\author{
Andréa Marcia Legnani ${ }^{1}$ \\ Mauro José Ferreira Cury ${ }^{2}$
}

\begin{abstract}
Resumo
O objetivo deste artigo consiste em analisar a integração cultural que acontece nas práticas educativas da Escola Latino Americana de Agroecologia (ELAA). Pretende contribuir com o debate acerca da integração latinoamericana, ampliando o campo de conhecimento sobre o tema. A pesquisa caracteriza-se como de abordagem qualitativa, sendo, a primeira fase, de pesquisa exploratória, bibliográfica e documental, e, a segunda fase, o estudo de caso do tipo exploratório e descritivo, tendo como instrumento de coleta de dados a entrevista semiestruturada. A integração cultural, na perspectiva da ELAA, acontece na troca de conhecimentos entre os educandos: cada um fala, ensina sobre a cultura de seu país, de sua comunidade e também conhece a dos outros países. Reconhece-se na sua cultura e conhece a cultura do outro num processo de respeito e valorização cultural dos diferentes países latino-americanos. Busca-se uma concepção de integração cultural tomada como um processo social dos povos latino-americanos, pelo estabelecimento de uma sociedade única e ao mesmo tempo diversa, sem fronteiras geográficas e livres de qualquer tipo de preconceito.
\end{abstract}

Palavras-chave: Integração Cultural ; Fronteira; Território; Territorialidade.

\section{Resumen}

El objetivo de este artículo consiste en analizar la integración cultural que ocurre en las prácticas educativas de la Escuela Latinoamericana de Agroecología (ELAA). Pretende contribuir con el debate sobre la integración latinoamericana, ampliando el campo de conocimiento sobre el tema. La investigación se caracteriza como de abordaje cualitativo, siendo, la primera fase, de investigación exploratoria de carácter bibliográfico y documental y, la segunda fase, teniendo como estrategia metodológica el estudio de caso del tipo exploratorio, descriptivoy, como instrumento de recolección, la entrevista semiestructurada. La integración cultural, en la perspectiva de la ELAA, ocurre en el intercambio de conocimientos entre los educandos: cada uno habla, enseña sobre la cultura de su país, de su comunidad y también conoce la de los otros países. Se reconoce en su cultura y conoce la cultura del otro en un proceso de respeto y valorización cultural de los diferentes países latinoamericanos. Una concepción de integración cultural tomada como un proceso social de los pueblos latinoamericanos, por el establecimiento de una sociedad única y al mismo tiempo diversa, sin fronteras geográficas y libres de cualquier tipo de prejuicio.

Palabras claves:La integración cultural; Frontera; Territorio; Territorialidad.

1 Mestre em Sociedade Cultura e Fronteiras; Aluna Especial da disciplina Territórios, Territorialidades, Poder, Fronteiras e Redes do Programa de Programa de Pós-Graduação Stricto Sensu - SOCIEDADE, CULTURA E FRONTEIRAS UNIOESTE; Foz do Iguaçu, Paraná e Brasil; andrea.legnani @ hotmail.com

2 Pós Doutorado em Geografia na Universidade Federal do Paraná, UFPR. E em Patrimônio e Turismo Cultural pela Universitat de Barcelona, Bolsista CAPES. Doutor em Geografia pela Universidade Federal do Paraná. UFPR. Professor Adjunto C da Universidade Estadual do Oeste do Paraná - UNIOESTE, Campus de Foz do Iguaçu. Docente dos Programas de Strictu-sensu em Geografia (Mestrado) da UNIOESTE - Campus de Marechal Cândido Rondon e Sociedade, Cultura e Fronteiras (Mestrado e Doutorado) da UNIOESTE - Campus de Foz do Iguaçu. Líder do Grupo de Pesquisa Turismo e Hospitalidade CNPq. E-mail: maurojfc@ gmail.com 


\begin{abstract}
The objective of this article is to analyze the cultural integration that takes place in the educational practices of the Latin American School of Agroecology (ELAA). It intends to contribute to the debate on Latin American integration, expanding the field of knowledge on the subject. The research is characterized as a qualitative approach, being the first phase of exploratory, bibliographic and documentary research, and the second phase, the exploratory and descriptive case study, having as instrument of data collection the semi-structured interview - Cultural integration, from the perspective of ELAA, takes place in the exchange of knowledge among the students: each one speaks, teaches about the culture of his country, his community and also knows the culture of other countries. It recognizes itself in its culture and knows the culture of the other in a process of respect and cultural appreciation of the different Latin American countries. It seeks a conception of cultural integration taken as a social process of the Latin American peoples, by the establishment of a unique and at the same time diverse society, without geographical borders and free of any kind of prejudice.
\end{abstract}

Keywords: Cultural Integration; Border; Territory; Territoriality.

\title{
1. Introdução
}

Este texto é parte da pesquisa desenvolvida durante a realização do mestrado, no Programa de Pós-Graduação Sociedade Cultura e Fronteiras, e aborda a temática da integração cultural na perspectiva da Escola Latino-Americana de Agroecologia (ELAA). Tem como objetivo analisar a integração cultural que acontece nas práticas educativas da ELAA.

Os estudos sobre integração da América Latina geralmente estão focados no âmbito econômico; são projetos integracionistas que blocos de países buscaram colocar em prática no decorrer da história, como o MERCOSUL, IIRSA e ALBA-TCP. Inerente à construção destes processos, emergem outros, com propostas que trazem uma concepção de integração diferenciada, como é o caso dos movimentos sociais.

A partir da década de 1990, distintos movimentos sociais eclodiram na América Latina. Muitos desses movimentos já existiam antes desta década, mas passaram a ter mais visibilidade e expressividade em decorrência de suas ações e reivindicações, sendo conhecidos internacionalmente (NOGUEIRA, 2011). Dentre esses movimentos, destaca-se o Movimento dos Trabalhadores Rurais Sem Terra (MST) (NOGUEIRA, 2011).

O MST, ao longo de sua trajetória, vem ampliando sua pauta de reivindicações, para além da questão da terra, passando pelo acesso à educação e desenvolvimento de ações relacionadas à integração latino-americana. A Escola Latino Americana de Agroecologia (ELAA) é uma dessas ações.

Quando o tema envolve a integração entre países é importante trazer para contextualização os conceitos de território, territorialidade e fronteira para compreender as possibilidades de uma integração cultural e de como ela pode acontecer. 
O território origina-se a partir do espaço, que é resultado de ação conduzida por um ator, que ao se apropriar do espaço, concreta ou abstratamente "territorializa" este espaço (RAFFESTIN, 1993).

A territorialidade é reflexo da multidimensionalidade do "vivido" territorial pela coletividade por meio de relações existenciais ou produtivas. Essas relações são de poder, pois acontece na interação entre os atores que buscam modificar, tanto relações com a natureza, como as relações sociais (RAFFESTIN, 1993).

Em relação à fronteira, segundo Ricardo José Batista Nogueira (2007, p. 29), "as origens políticas do conceito estão associadas à própria formação dos Estados-nacionais, que no seu processo de consolidação tiveram, e ainda tem que demarcar claramente as linhas divisórias [...]". O conceito de fronteira é resultado de uma construção histórica, um divisor de soberanias, está associado a disputas de poder, defesa de territórios e limite das leis (CURCIO, 2014).

Entretanto, a ideia de fronteira orientadora neste estudo, excede as suas definições tradicionais, numa perspectiva mais abrangente, envolvendo as representações culturais, que, por sua vez, "remetem à vivência, às socialidades, às formas de pensar intercambiáveis, aos ethos, valores, significados contidos nas coisas, palavras, gestos, ritos, comportamentos e ideias" (PESAVENTO, 2002, p.36). As fronteiras determinam limites e constituindo-se em linha que delimita os espaços, a fronteira é "ambivalente porque comporta dois estados de ser, e ambíguo porque traz consigo uma promessa de superação no tempo, na possibilidade de ser um outro, um terceiro" (PESAVENTO, 2002, p.37).

A integração cultural ultrapassa as fronteiras geográficas e os territórios formando uma nova territorialidade, com significados próprios sem sobrepor uma cultura sobre a outra, construindo uma integração na troca de experiências e conhecimentos.

Para apresentar os resultados da pesquisa o artigo está dividido em três partes; a primeira irá tratar do histórico da Escola Latino Americana de Agroecologia (ELAA) e sua dinâmica. A segunda parte apresenta a metodologia utilizada para atingir o objetivo da pesquisa. A terceira parte trata da análise e discussão dos resultados baseado nas entrevistas realizadas no campo de estudo. Finalizando o artigo são elencadas algumas considerações sobre a perspectiva de integração cultural presente nas práticas educativas da ELAA. 


\section{A Escola Latino Americana de Agroecologia}

A ELAA tem suas origens durante a realização do V Fórum Social Mundial realizado em 2005, na cidade de Porto Alegre - RS. É resultado de um protocolo de intenções e compromissos, firmado entre a Via Campesina, Governo da Venezuela, Governo do Brasil, Governo do Estado do Paraná e instituições de ensino do Brasil e da Venezuela (PROTOCOLO DE INTENCIONES, 2005).

A ELAA está localizada do Assentamento Contestado, no Município da Lapa Paraná, distante 60 km de Curitiba, onde, desde 1999, estão assentadas 108 famílias.

Ressaltando que o Contestado é um assentamento que, desde seu início, foi discutido por meio dos princípios da agroecologia e da cooperação, tratando-se de um grande laboratório para as aulas práticas (ELAA, 2011).

Em parceria com o Instituto Federal do Paraná (IFPR) ${ }^{3}$ um dos objetivos da ELAA é formar tecnólogos de nível superior que tenham a capacidade de: 1) organizar banco de sementes em suas regiões, em todos os países; 2) desenvolver e aplicar novas tecnologias agrícolas que respeitem o meio ambiente sem uso de agrotóxicos, nem adubos químicos solúveis; 3) reproduzir e ampliar os conhecimentos nas organizações camponesas às quais estão vinculados; 4) organizar e orientar os camponeses e camponesas para serem multiplicadores de sementes em suas regiões; 5) criar uma base técnica homogênea em todo o continente para aplicação de novas técnicas agrícolas em defesa das sementes crioulas (IFPR, 2006).

A intenção dessa Escola é envolver diretamente jovens camponeses para que participem na organização da produção, da cooperação e ações de preservação e conservação ambiental, nas diversas organizações campesinas da América Latina.

A ELAA iniciou suas atividades em 27 de agosto de 2005, com a primeira turma Mata Atlântica, que formou os primeiros 22 Tecnólogos em Agroecologia. Em 2006, iniciou-se a segunda turma, Resistência Camponesa, constituída de educandos de 18 Estados do Brasil e

\footnotetext{
${ }^{3}$ Com a Lei no 11.892/2008 de criação dos Institutos Federais, a escola Técnica da UFPR foi desmembrada da Universidade, e passou a ser IFPR. Atualmente é o IFPR que regulamenta o curso de Tecnologia em Agroecologia, enquanto instituição de ensino, e todos os outros cursos técnicos em agroecologia em parceria com os movimentos sociais no Estado do Paraná.
} 
do Paraguai. A terceira turma iniciou as aulas em abril de 2010, composta por 65 estudantes oriundos de vários países da América, dentre eles, além do Brasil, o Haiti, República Dominicana, Equador, Paraguai e da Colômbia (LIMA, 2011).

A terceira turma, denominada Semente Latina chegou ao final do curso com 50 educandos. A formatura aconteceu no dia 25 de outubro de 2013 com 49 formandos.

A turma Semente Latina faz parte da trajetória da pesquisa, pois era a turma que estava em andamento quando da primeira visita a ELAA em outubro de 2011 e na qual foram realizadas as entrevistas em 2013.

A Escola atende educandos dos Movimentos Sociais do Campo articulados à Via Campesina da América Latina e oferece o Curso Superior de Tecnologia em Agroecologia.

Para ingressar no curso, os candidatos devem atender aos seguintes critérios: ser indicado, por meio de carta enviada ao setor pedagógico, por movimento social do campo articulado na Via Campesina; ser militante do movimento que o indicou; ter disponibilidade para participar do curso, durante 3 anos e meio; ter idade mínima de 18 anos; ter perspectiva de crescimento na organização e luta dos movimentos sociais; participação em atividades preparatórias ao curso, organizadas e desenvolvidas pelas entidades/organizações mantenedoras do curso e comprovar estado de saúde que permita a realização do curso (ELAA, 2011).

Esse curso foi elaborado com base em diversas experiências já acumuladas pelos movimentos sociais participantes da Via Campesina, com destaque especial ao MST, por ser o Movimento que mais desenvolveu experiências de educação formal nos acampamentos e assentamentos do país.

O curso de Tecnologia em Agroecologia utiliza a pedagogia da alternância ${ }^{4}$ que alterna dois momentos educativos: o Tempo Escola e o Tempo Comunidade ${ }^{5}$. No Tempo Escola os educandos aprendem e desenvolvem o aprofundamento do conhecimento teórico, apoiados e assessorados por educadores de diferentes áreas do conhecimento; ao mesmo tempo em que desenvolvem vários trabalhos e práticas agroecológicas junto às famílias do Assentamento Contestado. Durante o Tempo Escola ainda são desenvolvidas diversas atividades organizadas

\footnotetext{
${ }^{4} \mathrm{O}$ curso é realizado em módulos que duram aproximadamente 75 dias, o Tempo Escola, com espaços de aproximadamente 90 dias entre eles, o Tempo Comunidade (TARDIN, 2009).
} 
em tempos educativos: Tempo de Leitura, Tempo de Trabalho, Tempo de Socialização de Experiências, Tempo Esporte, Círculo da Cultura e Noite Cultural. Há também tempos educativos dedicados à autogestão da turma, num sistema de cogestão com a Coordenação Executiva da Escola, nos quais os educandos e educandas participam da organização do espaço e convivência durante o tempo escola (Id. Ibid.).

Os educandos são organizados em núcleos de base, os quais escolhem um coordenador e uma coordenadora que participam da Coordenação dos Núcleos de Base da Turma (CNBT), sendo dois coordenadores escolhidos para participar das reuniões da Coordenação Executiva da Escola. Também são organizadas equipes de trabalho de: Relações Humanas, Comunicação e Cultura, Esporte e Lazer, Higiene e Saúde, Relatoria e Memória. Os estudantes ainda participam das atividades de alguns setores de trabalho: Cozinha e Refeitório, Administrativo, Pedagógico, Infraestrutura e Produção (Id. Ibid.).

Retomando a descrição da organização do curso de Tecnologia em Agroecologia, remete-se, agora, ao Tempo Comunidade, que acontece nas comunidades de origem dos educandos e vinculados aos seus movimentos sociais. No período do Tempo Comunidade, eles desenvolvem diversas atividades do curso, como estágios, pesquisas recomendadas pelos educadores, sistematização e experiências agroecológicas, leituras orientadas e o Trabalho de Conclusão de Curso, bem como realizam atividades solicitadas pelo Movimento Social do qual fazem parte (Id. Ibid.).

A metodologia desenvolvida no Curso é denominada de "Diálogo dos Saberes", que tem como base o pensamento de Paulo Freire. O método é praticado por cada um dos educandos no transcorrer de todo o curso de Tecnologia em Agroecologia, durante o tempo Comunidade, junto às famílias das comunidades das quais estão inseridos. Nos períodos de Tempo Escola, são organizados em núcleos e desenvolvem cada qual, o Diálogo com uma família que vive no Assentamento Contestado (Id. Ibid.).

Analisando o projeto do curso nos pontos que tratam da América Latina, as primeiras impressões indicam que um dos objetivos é propiciar aos educandos, além da formação profissional, uma formação que impulsione o desenvolvimento de uma consciência integracionista, começando pelo conhecimento da história latino-americana, incentivando a participação ativa em suas comunidades e países de origem, na transformação da realidade latino-americana. Uma concepção de integração baseada na construção de um processo 
pensado a partir das necessidades dos povos, da soberania e bem-estar dos países latinoamericanos, tendo como principais protagonistas os educandos.

\section{Metodologia}

A metodologia consistiu na primeira fase, em consulta a fontes bibliográficas e acervo documental do MST/PR para suporte teórico a pesquisa.

Na segunda fase, trabalhou-se com o estudo de caso e, como instrumento de coleta de dados, com a observação direta, utilizando a entrevista semiestruturada, considerada por Yian (2001) como uma fonte essencial de evidências.

Após o parecer ( $n^{\circ}$ 063/2013) de aprovação do projeto de pesquisa pelo Comitê de Ética em Pesquisa com Seres Humanos - CEP da Universidade Estadual do Oeste do Paraná realizou-se contato com a Coordenação da ELAA para agendar uma visita e realizar as entrevistas.

As questões das entrevistas realizadas com educandos e responsáveis pela coordenação da ELAA estavam relacionadas com as práticas desenvolvidas na escola que envolve a questão da Integração da América Latina.

As entrevistas foram gravadas em áudio e realizadas em português. Apesar dos educandos estrangeiros terem como língua oficial o espanhol, falam e compreendem o português, sendo que, apenas em algumas vezes, utilizaram palavras em espanhol.

As questões das entrevistas realizadas com educandos e responsáveis pela coordenação da ELAA estavam relacionadas com as práticas desenvolvidas na escola que envolve a questão da Integração da América Latina. Desta forma, com o total de 50 educandos e educandas, sendo 35 (trinta e cinco) do Brasil, 6 (seis) do Paraguai, 5 (cinco) do Equador, 3 (três) da Colômbia e 1 (um) da República Dominicana, foram realizadas seis (6) entrevistas. Os entrevistados foram escolhidos de forma aleatória conforme a disponibilidade e o interesse de participação, sendo um (1) educando da República Dominicana, um (1) do Paraguai, um (1) do Equador, um (1) da Colômbia e dois (2) do Brasil. Em relação ao corpo diretivo da ELAA, foram entrevistados três (3) coordenadores, sendo 2 brasileiros e 1 colombiano.

Das entrevistas realizadas, foram selecionadas para análise neste estudo as práticas educativas que proporcionam uma integração cultural: Tempo Circulo da Cultura, noites culturais, mística, tempo formatura. 


\section{Resultados e Discussões}

As entrevistas foram realizadas nos dias 01 e 02 de setembro e 16 de outubro de 2013. Em setembro era período de finalização das bancas de Trabalho de Conclusão de Curso dos educandos e educandas. Por este motivo, estavam um pouco tensos e agitados, mas isso não prejudicou a realização das entrevistas.

A realização das entrevistas proporcionou informações e reflexões extremamente ricas, com depoimentos sobre atividades educativas realizadas na Escola que promovem a integração cultural.

A fala de todos os participantes da pesquisa destaca que, o conteúdo relacionado à cultura dos povos latino-americanos está presente em várias atividades educativas, ficando clara a importância que a escola dá ao tema.

O Círculo de Cultura é um tempo destinado à aprendizagem em processos coletivos que podem envolver socialização de conhecimentos, desenvolvimento de habilidades artísticas, através do artesanato, da música, da dança, da poesia, do teatro entre outros. Tempo destinado ao cultivo e reflexão das diversas expressões da cultura popular relacionadas a Identidade Camponesa, valorização da luta e dos símbolos da Classe Trabalhadora. Exibição de filmes. Mostra de filmes com temas relacionados ao conteúdo trabalhado nas etapas (Documento Orientador ELAA, Lapa, 2011).

As atividades desenvolvidas no Tempo Círculo da Cultura auxiliam na criação de uma identidade latino-americana.

O aprofundamento da identidade latino-americana certamente demanda outro estudo, por isso, a que se faz aqui apenas uma menção para compreender a contextualização do termo com relação à problematização que envolve o estudo da integração cultural.

Assim, nesta pesquisa, apresenta-se a definição posta nos estudos de Alves:

Neste trabalho, adotaremos então a definição de identidade como o posicionamento subjetivo do ser humano diante de uma realidade histórica e social que o leva a sentir que compartilha conteúdos simbólico-afetivos com outros indivíduos, compondo assim um grupo ao qual há um sentimento de pertença. Ao colocarmos o termo 'posicionamento' em nossa concepção pretendemos frisar dois aspectos: o primeiro é de que há necessariamente uma postura ativa do sujeito e o segundo é que se trata de uma configuração subjetiva dinâmica e nunca rígida, pois depende de fatores históricos, sociais e situacionais para emergir como identidade.

Por conteúdos simbólico-afetivos, entendemos elementos culturais, que, justamente por serem culturais, são simbólicos, ou seja, são construídos e carregados de um valor que é significado socialmente. Assim, podemos falar de valores, crenças, representações, normas, atribuições de sentidos, memórias, gostos, interesses, etc., e também de elementos mais concretos como língua, história, religião, ritos, hinos, datas comemorativas, personalidades, festas públicas, etc. Todos esses conteúdos 
simbólicos são carregados de afeto e de valor, atribuídos de forma distinta por cada indivíduo na cultura, a partir das vivências pessoais e sociais estabelecidas (ALVES, 2013, p. 28).

A pesquisadora complementa que entende "a identidade latino-americana como um posicionamento de identificação que se funda em sentimentos de pertença à região e de compartilhamento de conteúdos simbólico-afetivos entre seus habitantes" (ALVES, 2013, p.40).

Outra prática, que trabalha a questão da integração, destacada pelos educandos e coordenadores, são as noites culturais.

\footnotetext{
Na organização e realização das 'Noites Culturais' que são realizadas a cada sábado no período noturno. Esta atividade é planejada pela Equipe de Comunicação e Cultura conformada por representantes de cada NB (Núcleo de Base), e com variações ao longo do curso abordam vários aspectos culturais étnicos como alimentação, vestimentas, músicas e danças, artes plásticas, etc. Esta mesma Equipe se encarrega de organizar os momentos musicais, como pela manhã ou nos horários de refeições, assim como a Banda Sovaco de Cobra composta por educandos e educandas apresenta repertório musical latino-americano diverso (Entrevistado Coordenador Brasileiro $\mathrm{n}^{\circ}$ 02, 16/10/2013).
}

Soma-se a esta prática o Tempo Formatura, tempo diário do conjunto da ELAA destinado à motivação das atividades do dia, conferência das presenças por Núcleo de Base, informes gerais e cultivo da mística do cuidado individual e da coletividade, e fortalecendo a unidade como Via Campesina e Classe Trabalhadora, cultivando a Identidade camponesa Latino-Americana e o sentimento Internacionalista (Documento Orientador ELAA, Lapa, 2001, p. 13).

A Mística, atividade diária realizada na Escola, é um espaço aberto para o trabalho de integração cultural.

A intenção não é aprofundar a discussão conceitual do significado da mística, pois o entendimento é complexo dependendo da abordagem escolhida, o interesse aqui é compreendê-la sob o enfoque do MST.

Sob o enfoque da formação dos sem-terra, a reflexão sobre o sentido de vivenciar a mística envolve três aspectos: a relação entre a mística e a formação dos valores humanos que sustentam a escolha de continuar na luta, a relação da mística e o cultivo da história ou da memória do povo e a mística como experiência de produção cultural (CALDART, 2004). 


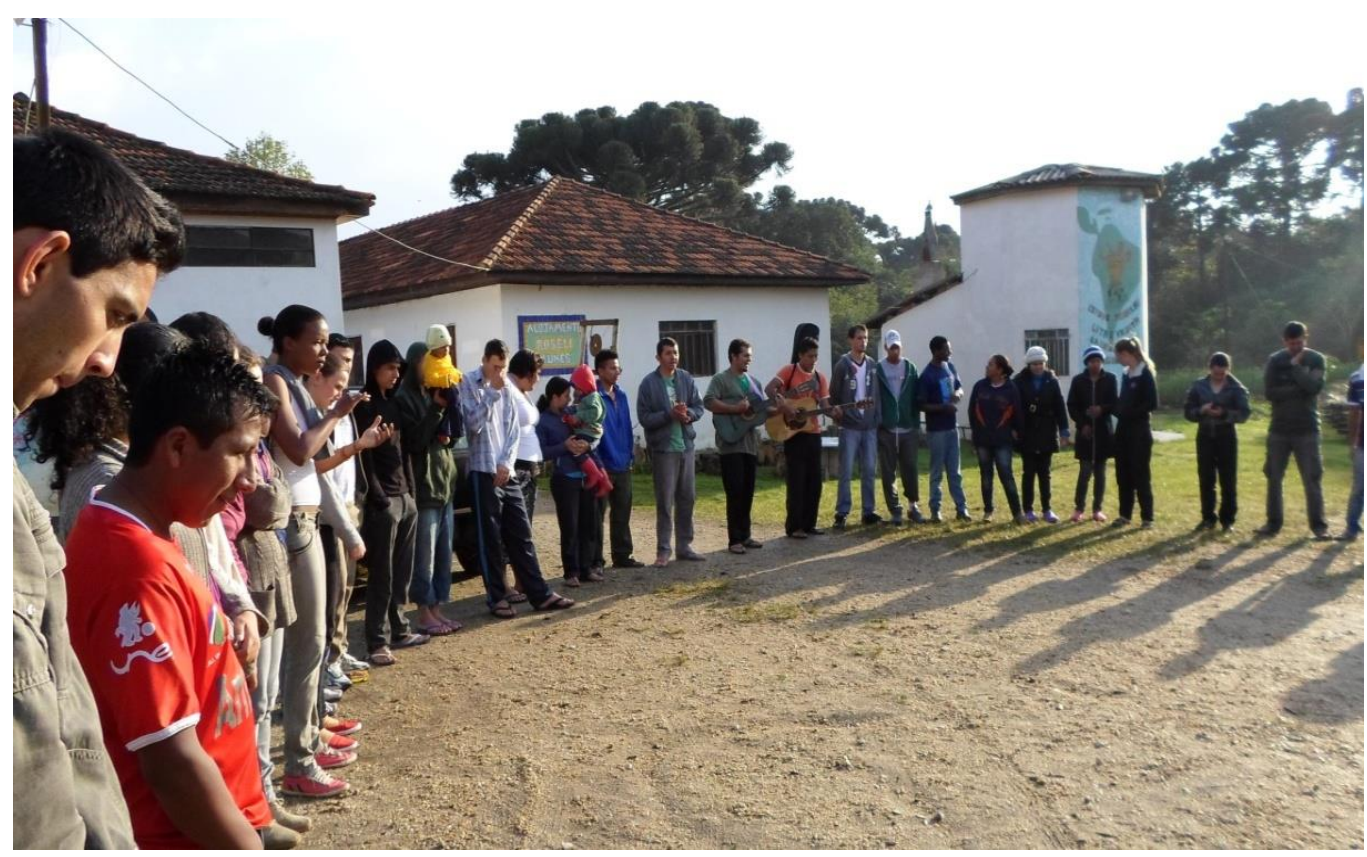

Fotografia 01: Tempo Formatura, cultivo da Mística

Ao analisar a questão da integração cultural e se a mesma é possível, diante da diversidade cultural da América Latina, pode-se perguntar conforme Clemente (1994, p.60) “Como é que a nossa geração, as próximas gerações, faremos para reunir, integrar povos tão diversos?".

Questão complexa para se responder. Mas, conforme Ricobom (2010, p.3745), “Mais do que buscar similaridades para justificar a necessidade de integração, é preciso reconhecer que a riqueza da América Latina está exatamente em sua diversidade cultural”.

Entre desafio e possibilidade de integração, a cultura pode ser analisada como fator importante nos processos de integração, destacado por Ricobom (2010, p.3746) que diz "O diálogo intercultural não se propõe a encontrar um acordo absoluto, que permita minimizar as diferenças, e que ao final se coloque como universal". E complementa ainda que "é preciso, pois, encontrar um critério que proíba a adoção de teorias ou práticas sociais como regras gerais, ou que permita a universalidade em sentido concreto e abstrato, ou seja, que não significa a imposição de determinada cultura”.

Mas, apesar da questão cultural da América Latina apresentar-se, de acordo com Souza (2012), como um desafio, como já citado anteriormente, a cultura pode ser vista como elemento de integração, como bem argumenta Veloso:

Entendemos que as manifestações culturais, estéticas e patrimoniais dos diferentes grupos sociais podem colaborar para a constituição de novos agenciamentos, de novas e renovadas identidades coletivas, viabilizando a reprodução social de diferentes grupos sociais [...], e possibilitando novas articulações entre cultura e desenvolvimento sustentável. Mas uma vez, a cultura pode conectar recursos 
simbólicos, econômicos e políticos. O que se deseja enfatizar é a importância dos muitos saberes existentes em nosso continente, a riqueza e a diversidade de nossa cultura e de nossa arte. [...] (VELOSO, 2008, p. 2-3).

Posta sob o prisma de elemento de integração, pensar a cultura como campo de possibilidades interativas e propositivas na organização dos interesses coletivos é uma tarefa necessária para todos os envolvidos no processo de integração (VELOSO, 2008).

Assim, entre desafio e possibilidade de integração, a cultura pode ser analisada como fator importante nos processos de integração, como destacado por Ricobom (2010, p. 3746) “ não com o objetivo de buscar a negação pela diferença, ou seja, sem sobreposição de culturas, mas sim pelo entrelaçamento dos variados aspectos culturais".

Neste sentido, a integração cultural, na perspectiva da ELAA, acontece na troca de conhecimentos entre os educandos: cada um fala, ensina sobre a cultura de seu país, de sua comunidade e também conhece a dos outros países. Reconhece-se na sua cultura e conhece a cultura do outro num processo de respeito e valorização cultural dos diferentes países latinoamericanos e Estados brasileiros.

Uma concepção de integração cultural tomada como um processo social dos povos latino-americanos, pelo estabelecimento de uma sociedade única e ao mesmo tempo diversa, sem fronteiras geográficas e livres de qualquer tipo de preconceito.

\section{Conclusões}

O referencial teórico proporcionou contextualizar o objeto de estudo e dar suporte para as análises das informações levantadas. A realização das entrevistas enriqueceu a pesquisa, porque foi possível um contato mais próximo com a realidade, com os sujeitos envolvidos, o que possibilitou esclarecer alguns pontos, confirmar algumas ideias perceber que não há verdades prontas e acabadas quando se propõe a realizar um estudo cientifico.

Esses elementos possibilitaram compreender a integração cultural da ELAA mais de perto, por meio aa integração entre os povos que acontece na própria convivência do dia a dia da Escola entre os educandos, no intercâmbio de culturas, conhecimentos e experiências proporcionados pelas atividades educativas.

As impressões ao final deste estudo apontam para uma concepção de integração cultural que está baseada na cooperação, na solidariedade e na integração dos povos, envolvendo as dimensões política, social, educacional e cultural em prol do desenvolvimento, da soberania e do bem estar dos povos da América Latina. 
É possível concluir que as práticas educativas da ELAA se materializam como um instrumento de promoção da integração cultural, proporcionada pelo acesso à educação, que inicia com a possibilidade de ingresso de participantes de movimentos sociais de países da América Latina, o que possibilita a troca de conhecimentos e experiências, valorização da cultura de cada povo.

\section{Referências}

ALVES. C. B. Integração, Identidade e Universidade na América Latina. Dissertação de Mestrado em Processos de Desenvolvimento Humano e Saúde. Universidade de Brasília, 2013. Disponível em: < repositorio.unb.br/bitstream/10482/13184/2013_CandidaBeatrizAlves.pdf > Acesso em: 21 out. 2013.

CALDART, R. S. Pedagogia do Movimento Sem Terra. 3. ed. São Paulo: Expressão Popular. 2004.

CLEMENTE, E. Integração: Língua, Cultura e Literatura. Porto Alegre: EDIPURS, 1994.

CURCIO, Daniela da Rosa. Cooperação e Integração na Fronteira Brasil-Uruguai Desafios e Perspectivas na Implantação de Cursos Binacionais de Educação Técnica. Anais do I Congresso Brasileiro de Geografia Política, Geopolítica e Gestão do Território, 2014.

ELAA. Escola Latino Americana de Agroecologia - ELAA: Documento Orientador, Lapa, PR: 2011.

NOGUEIRA, Ricardo José Batista. Fronteira: Espaço de Referência Identitária? Revista Ateliê Geográfico da Universidade Federal de Goiânia - UFG/IESA. V.1, n.2, Dez/2007, Goiânia-GO, 2007. Disponível em http://www.revistas.ufg.br/index.php/atelie/article/view/3013/3051. Acesso em: 04 Set. 2016.

NOGUEIRA, A. P. F. A luta dos movimentos sociais do campo na América Latina pelo acesso à educação: uma questão de autonomia. Anais da XII Jornada do Trabalho. Curitiba. 2011. Disponível em: <http://www4.fct.unesp.br/ceget/ANAISXII/GT3/Alexandre\%Peixoto\%20F,\%20Nogueira.pd f>. Acesso em: 09 mar. 2013.

PESAVENTO, Sandra Jatahy. Além das fronteiras. In: MARTINS, Maria Helena. (org.) Fronteiras Culturais: Brasil - Uruguai - Argentina. São Paulo: Ateliê Editorial, 2002.

RAFFESTIN, C. Por uma Geografia do Poder. Tradução Maria Cecília França. São Paulo: Editora Ática. 1993.

RICOBOM, G. A Integração Latino-Americana e o Diálogo Intercultural: Novas Perspectivas a Partir da Universidade. In: XIX Encontro Nacional da CONPEDI. Florianopolis: Fundação Boiteux, 2010. Anais eletrônicos. Disponível em: < http://www.conpedi.br/manaus/arquivos/anais/fortaleza/3573.pdf > Acesso em: 8 nov. 2013.

YIAN, R. K. Estudo de Caso: Planejamento e Métodos. 2a ed. Porto Alegre: Bookman, 2001. 
RELACult - Revista Latino-Americana de Estudos em Cultura e Sociedade

Revista Latinoamericana de Estudios en Cultura y Sociedad | Latin American Journal of Studies in Culture and Society V. 03, ed. especial, dez., 2017, artigo $\mathrm{n}^{\circ}$ 466 | relacult.claec.org |e-ISSN: 2525-7870 